Punjab University Journal of Mathematics (2021),53(6),409-424

https://doi.org/1.52280/pujm.2021.530604

\title{
An extension of TOPSIS based on linguistic terms in triangular intuitionistic fuzzy structure
}

\author{
Muhammad Saeed, Asad Mehmood and Amna Anwar \\ Department of Mathematics, University of Management and Technology, Lahore, \\ Pakistan, \\ Email: muhammad.saeed@umt.edu.pk*, a.asadkhan.khi@gmail.com, \\ amnaanwar5566@gmail.com
}

Received: 24 February, 2021 / Accepted: 03 June, 2021 / Published online: 25 June, 2021

\begin{abstract}
Chen [24] introduced the extension of TOPSIS in the fuzzy structure, while this article stretches the modern approach of TOPSIS to the intuitionistic fuzzy framework. Linguistic terms are used in this study to evaluate the weight of each criterion and the rating of alternatives in the context of a triangular intuitionistic fuzzy number. A new intuitionistic fuzzy positive ideal solution (IFPIS) and intuitionistic fuzzy negative ideal solution (IFNIS) are proposed in this model of extended TOPSIS. Euclidean distance is introduced between two triangular intuitionistic fuzzy numbers to calculate separation between each alternative to both (IFPIS) and (IFNIS). The proposed model's mechanism is presented with the help of an algorithm, and then it is applied to the personal selection problem. Finally, a comparative study is given between this model and other TOPSIS techniques.
\end{abstract}

Key Words: Extension of TOPSIS, Triangular Intuitionistic Fuzzy Number (TIFN), Linguistic Variable, Distance Measure.

\section{INTRODUCTION}

Several complicated models have been developed in recent years in the fields of technology, climate, research, society, and numerous other areas. To investigate problems like uncertainties and several other theories that are well established. These theories include a fuzzy set, intuitionistic fuzzy set, interval-valued intuitionistic fuzzy set ([35]-[2]), three of the most common theories. Usually, we naturally have a flippant type of difficulty in everyday life. When describing something, we use verbal or numerical terms, an event summary, order, and fugacity seen in many other cases. Men would not use terms to demonstrate confidence when negotiating a situation, only settle on a condition. It is called in the West, young, very old, very recent, depending on the person's age, teenage. The car is based on the road's slopes and ramps the action with the gas brake lever or pressure brake lever is steadily slower or progressively faster. The idea of fuzzy sets was presented by Zadeh [35]. 
From that point forward, the fuzzy sets and fuzzy rationale are utilized generally in numerous applications, including unpredictability. In any case, it is seen that there still stay a few circumstances that can't be secured by fuzzy sets; thus, the idea of interim esteemed fuzzy sets came into power to catch those circumstances. Although the Fuzzy set hypothesis effectively takes care of unpredictability emerging from obscurity or fractional acceptance of a component in a set, it can't demonstrate a wide range of unpredictability winning in diverse genuine physical issues. For example, issues including fragmented data. These are also examples of how man's consciousness acts under situations that are markedly uncertain and how events are interpreted, identified, and controlled. Zadeh developed and published the Fuzzy Set Theory (FS), which uses Fuzzy Logic principles, under which the study of complex structures added a new dimension in an initial 1965 paper [35]. Further, speculation of the fuzzy set was developed by Atanassov [1], and it is known as intuitionistic fuzzy sets (IFS). In IFS, rather than one participation grade, there is likewise a non-enrollment grade joined with every component. Further, there is a limitation that the entirety of these two evaluations is less or equivalent to solidarity. The origination of IFS can be seen as a fitting/elective methodology on the off chance that where accessible data isn't adequate to characterize the inaccuracy by the customary fuzzy sets. Atanassov later expanded intuitionistic fuzzy sets, and Gargov [2] to interim quality interval-value intuitionistic fuzzy sets. The membership functions are known as FS. These membership functions are, in fact, just fluctuating numbers. Dubois identified fuzzy numbers (FNs) and Prade [8] as a fuzzy subset of the genuine line. Fuzzy numbers allow one to transform linguistic variables in a fuzzy behavior into a numerical model. An FN is an amount whose worth is loose, as opposed to correct as for the situation with "normal" numbers. Any FN may be the conviction of as a capacity whereby space is a described set. Garge et al. [11] work explore many of the series of triangular numbers, abbreviated as T-numbers are amazingly rich in products. [31] He was the Yong-jie who describes certain simple operations, including addition, fuzzy multiplication of numbers, and Fuzzy Number Force of an intuitive fuzzy triangular number. Also, they explained certain suggestions about the rules and properties of these operations. A triangular intuitionistic fuzzy number (TIFN) was further described by Seikh et al. [27] to be approximated to a nearly approximate interval value. The implementation of this result and interval arithmetic solves a boundless optimization problem whose coefficients are TIFN set. Burillo [5] introduced the ranking methodology of Intuitionistic fuzzy numbers (IFNs) and contemplated annoyances of (IFN) and the first effect of the interrelation of these numbers. Number-crunching activities of the proposed IFN are assessed. Li et al. [17] describe the ranking system of triangular intuitionistic fuzzy numbers (TIFNs) as an integral challenge. And develop a method for solving the meaning and uncertaintybased ranking by TIFN. Further, evaluate arithmetic operations and split sets over TIFN. The index-value and index-ambiguity functions as well. The additional classification was introduced to solve multi-attributes' decision-making problems in which alternate attribute scores are represented using TIFNs. Nehi [21] introduced a new ranking method to rank intuitionistic fuzzy numbers. [36] Linguistic variables are central to manipulations of fuzzy logic, but are often overlooked in discussions about the validity of fuzzy logic. Santiago et al. [26] suggests a paradigm for linguistic variables and mechanism of fuzzyfication for fuzzy structures that discusses various degrees of ambiguity within the same linguistic variables. Also systems of Type-n with homogeneous linguistic variables. Later on, numerous 
techniques for MADM were presented, including the augmenting deviation technique [30], the TOPSIS strategy ([37],[32]), and the Elected technique [10]. Qin et al. [22] describes the multi-attribute collective decision-making (MAGDM) two essential tasks are to define the values of the attributes and produce a ranking of all alternatives. Linguistic variable based interval-valued intuitionistic fuzzy number is a superior method for the first task and aggregation operator is an efficient tool for the second task. The abbreviation TOPSIS represents a procedure for inclination by comparability to the perfect arrangement. TOPSIS was at first introduced by Hwang et al. [34] and then Lai et al. [16] likewise depicted the TOPSIS idea, alluding to positive and negative arrangements as the perfect and hostile to perfect arrangements, respectively. TOPSIS is alluring in that constrained emotional info is required from chiefs. Chen et al. [14] studied the analysis of weights of criteria for the multi-attributive decision making problems. TOPSIS has been applied to various applications ([15],[38]) even though it isn't so broadly applied as other multi-attribute techniques. The TOPSIS technique thinking about both positive-perfect and negative-perfect arrangement is only the mainstream strategies in various-property dynamic issue. In this way, TOPSIS technique joined with intuitionistic fuzzy numbers has a tremendous possibility of progress for the provider determination process. Chen [6] introduced the extension of TOPSIS to solve multiple criteria decision-making problems (MCDM) in triangular fuzzy number on the basis of linguistic variables. Chu et al. [7] has overcome with improvement in Chen's model. Atalik et al. ([4],[3]) proposed some rankings among triangular intuitionistic fuzzy numbrers based on gerogonne point and incircle of triangluar intuitionistic fuzzy number. Ghaemi et al. [12] applied TOPSIS technique on type-2 fuzzy set. Mahdavi et al. [18] proposed new extension of fuzzy TOPSIS and have the same results with Chen and Chu, while Gautam et al. [13] introduced the extension of TOPSIS in intuitionistic fuzzy structure. As per the chance hypothesis by Fuller and Majlender [9], it characterizes the weighted plausibility attitudinal expected estimations of TIFNs and subsequently presents another hazard attitudinal positioning strategy for TIFNs. This positioning strategy can adequately think about DMs' hazard demeanor and make the results progressively reliable with genuine circumstances. The affect-ability investigations on attitudinal character parameters are likewise given [33]. Saeed et al. ([23]-[28]) applied the generalized TOPSIS technique on soft and hypersoft structures, and also predicted the winner of FIFA World Cup 2018. Saqlain et al. [29] applied TOPSIS to predict the winner of Cricket World Cup 2019. Riaz et al. $[19,20]$ applied TOPSIS technique on the novel structure of Pythagorean $\mathrm{m}$-polar fuzzy set for the selection of advertisement mode.

1.1. Motivation. Many articles have been published on MCDM using the TOPSIS method, as different authors applied this method on fuzzy set, intuitionistic fuzzy set, neurotrophic set, soft set, and their hybrid structures. The concept of extension of TOPSIS based on linguistic variable in triangular intuitionistic fuzzy number is a modification of other TOPSIS extensions. This proposed technique evaluates the data in TIFN based on linguistic variables, making the decision-making process more appropriate and easy for the decisionmakers committee. Whereas the data can be evaluated in more depth in intuitionistic fuzzy structure than the fuzzy structure due to membership and non-membership degree. Here, a set of questions arises about how a TOPSIS can be applied on TIFN? What should be the normalization procedure for such an extension? How IFPIS and IFNIS can be defined? 
The distance measure formula for each alternative to IFPIS and IFNIS while considering the data in triangular intuitionistic fuzzy numbers. From this point of view, this extension of TOPSIS is more generalized and can be a good choice for a decision-maker in MCDM problems.

1.2. The Paper Presentation. In this article, the TOPSIS model is extended to the triangular intuitionistic fuzzy environment.

- Formulation of distance between two TIFNs

- Linguistic terms based TOPSIS technique

- Novel approach of IFPIS and IFNIS

- A case study with an algorithm

1.3. Structure of Paper. In section 2, Basic mathematical definitions related to proposed study are revised. The proposed model of extended TOPSIS is analyzed in section 3 , while section 4 deals with a case study problem correspond to desired idea. Further the results are related with five different articles in comparative approach as section 5. In the last section, a conclusion of the article is depicted.

\section{PRELIMINARIES}

In this section, we review some basic notions associated with the proposed article.

Definition 2.1. [1] Consider a universal set $Y$. An Intuitionistic fuzzy set $A$ in universe $Y$ can be defined as follows

$$
A=\left\{\left(y, \mu_{A}(y), \nu_{A}(y)\right): y \in Y\right\},
$$

where these function $\mu_{A}: Y \rightarrow[0,1]$ and $\nu_{A}: Y \rightarrow[0,1]$ define the degree value of membership and non-membership of the element $y \in Y$, respectively. For all $y \in Y: 0 \leq$ $\mu_{A}(y)+\nu_{A}(y) \leq 1$ and if $\pi_{A}(y)=1-\mu_{A}(y)-\nu_{A}(y)$, then $\pi_{A}(y)$ is the hesitancy degree of the element $y \in Y$ to the set $A$ and $\pi_{A}(y) \in[0,1]$, for all $y \in Y$

Definition 2.2. [21] An Intuitionistic Fuzzy Set is called convex Intuitionistic fuzzy set if and only if

$$
\begin{gathered}
\mu_{A}\left(\gamma y_{1}+(1-\gamma) y_{2}\right) \geq \min \left\{\mu_{A}\left(y_{1}\right), \mu_{A}\left(y_{2}\right)\right\} \text { and } \\
\nu_{A}\left(\gamma y_{1}+(1-\gamma) y_{2}\right) \leq \max \left\{\nu_{A}\left(y_{1}\right), \nu_{A}\left(y_{2}\right)\right\} \\
\text { where } \gamma \in[0,1] ; \text { for all } y_{1}, y_{2} \in Y
\end{gathered}
$$

Definition 2.3. [21] An Intuitionistic Fuzzy Set is called normal Intuitionistic fuzzy set, if there does have at the minimum two points $y_{1}, y_{2} \in Y$ such that $\mu_{A}\left(y_{1}\right)=1$ and $\nu_{A}\left(y_{2}\right)=0$.

Definition 2.4. [21] An Intuitionistic fuzzy number (IFN) $A$ is a subset of IFS over universal set $Y$ that is defined as

(1) A is convex IFS,

(2) A is normal IFS,

(3) $\mu_{A}$ is upper semi-continuous and $\nu_{A}$ is lower semi-continuous,

(4) Sup $A=\left\{y \in Y: \nu_{A}(y)<1\right\}$ is bounded. 
Definition 2.5. [13] $A$ Triagnular intuitionistic fuzzy number (TIFN) $\tilde{A}=\left(\left(\phi_{1}, \phi_{2}, \phi_{3} ; w_{A}\right),\left(\psi_{1}, \psi_{2}, \psi_{3} ; u_{A}\right)\right)$ is an IFS on the real number set $R$, where the membership function and non-membership function of $\tilde{A}$ are given as

$$
\widetilde{\mu_{A}}(y)= \begin{cases}0, & y<\phi_{1} \\ \frac{\left(y-\phi_{1}\right) w_{A}}{\phi_{2}-\phi_{1}}, & \phi_{1} \leq y<\phi_{2} \\ w_{A}, & y=\phi_{2} \\ \frac{\left(\phi_{3}-y\right) w_{A}}{\phi_{3}-\phi_{2}}, & \phi_{2}<y \leq \phi_{3} \\ 0, & y>\phi_{3}\end{cases}
$$

and

$$
\widetilde{\nu_{A}}(y)= \begin{cases}1, & y<\psi_{1} \\ \frac{\psi_{2}-y+u_{A}\left(y-\psi_{1}\right)}{\psi_{2}-\psi_{1}}, & \psi_{1} \leq y<\psi_{2} \\ u_{A}, & y=\psi_{2} ; \\ \frac{y-\psi_{2}+u_{A}\left(\psi_{3}-y\right)}{\psi_{3}-\psi_{2}}, & \psi_{2}<y \leq \psi_{3} \\ 1, & y>\psi_{3}\end{cases}
$$

respectively, where the values $\widetilde{\mu_{A}}$ and $\widetilde{\nu_{A}}$ represent the maximum value of membership and the minimum value of non-membership respectively, such that they satisfy the condition that is $0 \leq \widetilde{\mu_{A}}+\widetilde{\nu_{A}} \leq 1$. The graphical representation of TIFN is shown in Figure 1.

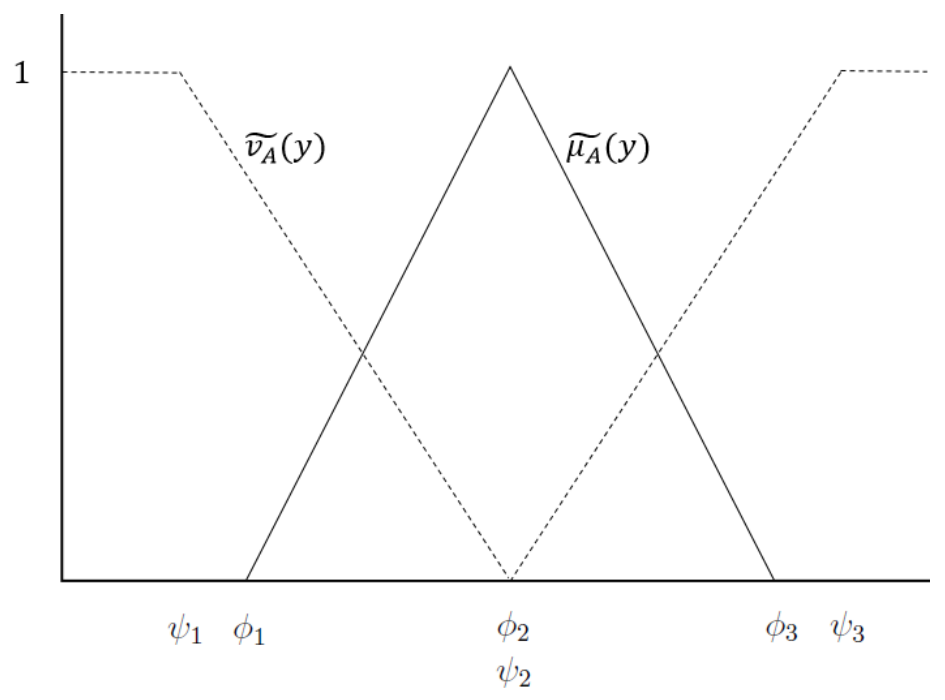

FIGURE 1. Triangular Intuitionistic Fuzzy Number

Proposition 2.6. [13] Let $\tilde{a}_{1}=\left(\left(\phi_{11}, \phi_{12}, \phi_{13}\right),\left(\psi_{11}, \psi_{12}, \psi_{13}\right)\right)$ and $\tilde{a}_{2}=\left(\left(\phi_{21}, \phi_{22}, \phi_{23}\right),\left(\psi_{21}, \psi_{22}, \psi_{23}\right)\right)$ be two triangular intuitionistic fuzzy number, if $\tilde{a}_{1}=\tilde{a}_{2}$ then $\phi_{11}=\phi_{21} ; \phi_{12}=\phi_{22}$; $\phi_{13}=\phi_{23} ; \psi_{11}=\psi_{21} ; \psi_{12}=\psi_{22} ;$ and $\psi_{13}=\psi_{23}$.

Definition 2.7. [36] Linguistic terms that represent certain variables, which further can be evaluated in numeric values are called linguistic variables (LV). 
Definition 2.8. [13] Let $\tilde{a}_{1}=\left(\left(\phi_{11}, \phi_{12}, \phi_{13}\right),\left(\psi_{11}, \psi_{12}, \psi_{13}\right)\right)$ and $\tilde{a}_{2}=\left(\left(\phi_{21}, \phi_{22}, \phi_{23}\right),\left(\psi_{21}, \psi_{22}, \psi_{23}\right)\right)$ be two triangular intuitionistic fuzzy numbers then vertex method (based on Euclidean distance) is defined for distance between TIFNs as

$$
\begin{aligned}
d\left(\tilde{a}_{1}, \tilde{a}_{2}\right)= & \left(\frac { 1 } { 6 } \left\{\left(\phi_{11}-\phi_{21}\right)^{2}+\left(\phi_{12}-\phi_{22}\right)^{2}+\left(\phi_{13}-\phi_{23}\right)^{2}+\right.\right. \\
& \left.\left.\left(\psi_{11}-\psi_{21}\right)^{2}+\left(\psi_{12}-\psi_{22}\right)^{2}+\left(\psi_{13}-\psi_{23}\right)^{2}\right\}\right)^{\frac{1}{2}}
\end{aligned}
$$

Theorem 2.9. The distance function from triangular intuitionistic fuzzy numbers (TIFNs) $\rightarrow R^{+} \cup\{0\}$ is a metric.

Proof:

Let $\tilde{a}_{1}, \tilde{a}_{2}$ and $\tilde{a}_{3}$ be three TIFNs, then

(1) $d\left(\tilde{a}_{1}, \tilde{a}_{2}\right) \geq 0$

(2) Suppose $d\left(\tilde{a}_{1}, \tilde{a}_{2}\right)=0$

$$
\begin{gathered}
\Leftrightarrow\left(\frac { 1 } { 6 } \left\{\left(\phi_{11}-\phi_{21}\right)^{2}+\left(\phi_{12}-\phi_{22}\right)^{2}+\left(\phi_{13}-\phi_{23}\right)^{2}\right.\right. \\
\left.\left.+\left(\psi_{11}-\psi_{21}\right)^{2}+\left(\psi_{12}-\psi_{22}\right)^{2}+\left(\psi_{13}-\psi_{23}\right)^{2}\right\}\right)^{\frac{1}{2}}=0 \\
\Leftrightarrow\left(\phi_{11}-\phi_{21}\right)^{2}+\left(\phi_{12}-\phi_{22}\right)^{2}+\left(\phi_{13}-\phi_{23}\right)^{2} \\
+\left(\psi_{11}-\psi_{21}\right)^{2}+\left(\psi_{12}-\psi_{22}\right)^{2}+\left(\psi_{13}-\psi_{23}\right)^{2}=0 \\
\left(\phi_{11}-\phi_{21}\right)^{2}=0 ; \quad
\end{gathered}
$$

(3) $d\left(\tilde{a}_{1}, \tilde{a}_{2}\right)=d\left(\tilde{a}_{2}, \tilde{a}_{1}\right)$

(4) For any three $\tilde{a}_{1}, \tilde{a}_{2}$ and $\tilde{a}_{3}$ TIFN

$$
\begin{gathered}
\left(\left(\phi_{11}-\phi_{21}\right)^{2}+\left(\phi_{12}-\phi_{22}\right)^{2}+\left(\phi_{13}-\phi_{23}\right)^{2}+\left(\psi_{11}-\psi_{21}\right)^{2}+\left(\psi_{12}-\psi_{22}\right)^{2}+\left(\psi_{13}-\psi_{23}\right)^{2}\right)^{\frac{1}{2}} \\
=\left(\left(\phi_{11}-\phi_{31}+\phi_{31}-\phi_{21}\right)^{2}+\left(\phi_{12}-\phi_{32}+\phi_{32}-\phi_{22}\right)^{2}+\left(\phi_{13}-\phi_{33}+\phi_{33}-\phi_{23}\right)^{2}\right. \\
\left.+\left(\psi_{11}-\psi_{31}+\psi_{31}-\psi_{21}\right)^{2}+\left(\psi_{12}-\psi_{32}+\psi_{32}-\psi_{22}\right)^{2}+\left(\psi_{13}-\psi_{33}+\psi_{33}-\psi_{23}\right)^{2}\right)^{\frac{1}{2}} \\
\leq\left(\left(\phi_{11}-\phi_{31}\right)^{2}+\left(\phi_{12}-\phi_{32}\right)^{2}+\left(\phi_{13}-\phi_{33}\right)^{2}+\left(\psi_{11}-\psi_{31}\right)^{2}+\left(\psi_{12}-\psi_{32}\right)^{2}+\left(\psi_{13}-\psi_{33}\right)^{2}\right)^{\frac{1}{2}} \\
+\left(\left(\phi_{31}-\phi_{21}\right)^{2}+\left(\phi_{32}-\phi_{22}\right)^{2}+\left(\phi_{33}-\phi_{23}\right)^{2}+\left(\psi_{31}-\psi_{21}\right)^{2}+\left(\psi_{32}-\psi_{22}\right)^{2}+\left(\psi_{33}-\psi_{23}\right)^{2}\right)^{\frac{1}{2}} \\
=d\left(\tilde{a}_{1}+\tilde{a}_{3}\right)+d\left(\tilde{a}_{3}+\tilde{a}_{2}\right)
\end{gathered}
$$


Thus,

$$
\Rightarrow d\left(\tilde{a}_{1}, \tilde{a}_{2}\right) \leq d\left(\tilde{a}_{1}+\tilde{a}_{3}\right)+d\left(\tilde{a}_{3}+\tilde{a}_{2}\right)
$$

This implies, defined distance function is a metric distance.

\section{Proposed Model}

The procedure of our proposed model of the extension of TOPSIS is studied in this section. This methodology is very applicable in group decision-making problems established on triangular intuitionistic fuzzy numbers (TIFNs). This methodology of TOPSIS on TIFN is the generalization of triangular fuzzy number (TFN), where we can deal the data in more depth with the membership function degree and non-membership function degree. The depth evaluation of the data gave more appropriate and better decision after the computation of TOPSIS.

In this article, linguistic variables (LV) are utilized for the weights of each criterion and the ratings of all alternatives correspond to each criterion. Further, Table 1 and Table 2 shows the linguistic variables (LV) in the expression of triangular intuitionistic fuzzy numbers (TIFNs).

\begin{tabular}{|l|l|}
\hline Very Low (VL) & $(0,0,0.1),(0,0,0.2)$ \\
\hline Low $(\mathrm{L})$ & $(0,0.1,0.3),(0,0.1,0.4)$ \\
\hline Medium Low $(\mathrm{ML})$ & $(0.1,0.3,0.5),(0.05,0.3,0.55)$ \\
\hline Medium (M) & $(0.3,0.5,0.7),(0.2,0.5,0.8)$ \\
\hline Medium High $(\mathrm{MH})$ & $(0.5,0.7,0.9),(0.45,0.7,0.95)$ \\
\hline High $(\mathrm{H})$ & $(0.7,0.9,1),(0.6,0.9,1)$ \\
\hline Very High $(\mathrm{VH})$ & $(0.9,1,1),(0.8,1,1)$ \\
\hline
\end{tabular}

Table $1: \mathrm{LV}$ for weights of criteria

\begin{tabular}{|l|l|}
\hline Very Poor (VP) & $(0,0,1),(0,0,2)$ \\
\hline Poor (P) & $(0,1,3),(0,1,4)$ \\
\hline Medium Poor (MP) & $(1,3,5),(0.5,3,5.5)$ \\
\hline Fair (F) & $(3,5,7),(2,5,8)$ \\
\hline Medium Good (MG) & $(5,7,9)(4.5,7,9.5)$ \\
\hline Good (G) & $(7,9,10),(6,9,10)$ \\
\hline Very Good (VG) & $(9,10,10),(8,10,10)$ \\
\hline
\end{tabular}

Table $2: \mathrm{LV}$ for rating of alternatives

The evaluation of weight for every criteria can be found by either pairwise comparisons or either directly assigning of weights [14]. In this paper, multiple decision makers give weights for each criteria $\operatorname{Cri}_{j}(j=1,2, \cdots, q)$ and the evaluate the ratings of all alternatives $\operatorname{Alt}_{i}(i=1,2, \cdots, p)$ corresponds to criteria defined in linguistic terms as shown in Table 1 and Table 2

Consider if there are $d$ decision makers, then ratings of alternatives and weights of each criteria can be calculated as 


$$
\begin{aligned}
\breve{g}_{i j} & =\frac{1}{d}\left[g_{i j}^{1}(+) g_{i j}^{2}(+) \cdots(+) g_{i j}^{d}\right] \\
\breve{w}_{j} & =\frac{1}{d}\left[w_{j}^{1}(+) w_{j}^{2}(+) \cdots(+) w_{j}^{d}\right]
\end{aligned}
$$

where $g_{i j}^{k}$ and $w_{j}^{k}$ are the ratings of each alternative and weights of each criteria associated by $k$ th decision maker.

After the calculation of group ratings of alternative and weights of criteria, then IF decision-making matrix can be expressed as follows

$$
\begin{gathered}
\breve{G}=\left[\begin{array}{cccc}
\breve{g}_{11} & \breve{g}_{12} & \cdots & \breve{g}_{1 q} \\
\breve{g}_{21} & \breve{g}_{22} & \cdots & \breve{g}_{2 q} \\
\vdots & \vdots & \ddots & \vdots \\
\breve{g}_{p 1} & \breve{g}_{p 2} & \cdots & \breve{g}_{p q}
\end{array}\right] \\
\breve{W}=\left\{\breve{w}_{1}, \breve{w}_{2}, \cdots, \breve{w}_{q}\right\}
\end{gathered}
$$

where $\breve{g}_{i j}$ (for all $i, j$ ) and $\breve{w}_{j},(j=1,2, \cdots, q)$ are in the form of linguistic variables and it is represented as

and

$$
\breve{g}_{i j}=\left(\left(\phi_{i j}^{(1)}, \phi_{i j}^{(2)}, \phi_{i j}^{(3)}\right),\left(\psi_{i j}^{(1)}, \psi_{i j}^{(2)}, \psi_{i j}^{(3)}\right)\right)
$$

$$
\breve{w}_{j}=\left(\left(\phi_{j}^{(1)}, \phi_{j}^{(2)}, \phi_{j}^{(3)}\right),\left(\psi_{j}^{(1)}, \psi_{j}^{(2)}, \psi_{j}^{(3)}\right)\right)
$$

in triangular intuitionistic fuzzy numbers.

To transform the various criteria scales into a comparable scales, the linear scale transformation is used. By this process, we get the normalized IF decision matrix and that is denoted as $\breve{R}$.

$$
\breve{R}=\left[\breve{r}_{i j}\right]_{p \times q}
$$

where,

$$
\breve{r}_{i j}=\left(\left(\frac{\phi_{i j}^{(1)}}{\phi_{j}^{(3) *}}, \frac{\phi_{i j}^{(2)}}{\phi_{j}^{(3) *}}, \frac{\phi_{i j}^{(3)}}{\phi_{j}^{(3) *}}\right),\left(\frac{\psi_{j}^{(1)-}}{\psi_{i j}^{(3)}}, \frac{\psi_{j}^{(1)-}}{\psi_{i j}^{(2)}}, \frac{\psi_{j}^{(1)-}}{\psi_{i j}^{(1)}}\right)\right),
$$

where $\phi_{j}^{(3) *}=\max \phi_{i j}^{(3)}$ and $\psi_{j}^{(1)-}=\min \psi_{i j}^{(1)} ;$ for $j \in B$

$$
\breve{r}_{i j}=\left(\left(\frac{\phi_{j}^{(1)-}}{\phi_{i j}^{(3)}}, \frac{\phi_{j}^{(1)-}}{\phi_{i j}^{(2)}}, \frac{\phi_{j}^{(1)-}}{\phi_{i j}^{(1)}}\right),\left(\frac{\psi_{i j}^{(1)}}{\psi_{j}^{(3) *}}, \frac{\psi_{i j}^{(2)}}{\psi_{j}^{(3) *}}, \frac{\psi_{i j}^{(3)}}{\psi_{j}^{(3) *}}\right)\right),
$$

where $\phi_{j}^{(1)-}=\min \phi_{i j}^{(1)}$ and $\psi_{j}^{(3) *}=\max \psi_{i j}^{(3)}$ for $j \in C$

The above method of normalization conserves the property of IFNs that is membership and non-membership of elements is lies between $[0,1]$.

A weighted normalized IF decision matrix denoted by $\breve{V}$ can be constructed as

$$
\breve{V}=\left[\breve{v}_{i j}\right]_{p \times q}
$$


where $\breve{v}$ can be calculated by considering the importance of weight of criteria as,

$$
\breve{v}_{i j}=\breve{r}_{i j}(\cdot) \breve{w}_{j}
$$

The elements $\breve{v}_{i j}$ (for all $i, j$ ) in weighted normalized IF decision matrix are normalized TIFNs and their membership and non-membership functions are in the interval $[0,1]$. Now, we can define intuitionistic fuzzy positive ideal solution (IFPIS), denoted by $A l t^{*}$ and intuitionistic fuzzy negative ideal solution (IFNIS), denoted by $A l t^{-}$as

where,

$$
\begin{gathered}
A l t^{*}=\left(\breve{v}_{1}^{*}, \breve{v}_{2}^{*}, \cdots, \breve{v}_{q}^{*}\right), \\
A l t^{-}=\left(\breve{v}_{1}^{-}, \breve{v}_{2}^{-}, \cdots, \breve{v}_{q}^{-}\right),
\end{gathered}
$$

$$
\breve{v}_{j}^{*}=\left\{\begin{array}{l}
(1,1,1),(0,0,0) \quad j \in B ; \\
(0,0,0),(1,1,1) \quad j \in C . \\
\quad \text { for all } j=1,2, \cdots, q
\end{array}\right.
$$

and

$$
\breve{v}_{j}^{-}=\left\{\begin{array}{l}
(0,0,0),(1,1,1) \quad j \in B ; \\
(1,1,1),(0,0,0) \quad j \in C . \\
\text { for all } j=1,2, \cdots, q
\end{array}\right.
$$

Then after, the Separation of each alternative from IFPIS $A l t^{*}$ and IFNIS $A l t^{-}$can be computed as

$$
\begin{gathered}
\breve{S}_{i}^{*}=\sum_{j=1}^{q} d\left(\breve{v}_{i j}, \breve{v}_{j}^{*}\right) \\
\breve{S}_{i}^{-}=\sum_{j=1}^{q} d\left(\breve{v}_{i j}, \breve{v}_{j}^{-}\right) \\
\quad \text { for all } i=1,2, \cdots, p
\end{gathered}
$$

where $d(\cdot, \cdot)$ is the distance measure between two TIFNs as stated above in (2.1).

Once the Separation of all alternatives $A l t_{i},(i=1,2, \cdots, p)$ are calculated, then closeness coefficient can be defined to rank the alternatives as

$$
\text { C.Coef } f_{i}=\frac{\breve{S}_{i}^{-}}{\breve{S}_{i}^{-}+\breve{S}_{i}^{*}}
$$

After the computation of the closeness coefficient C.Coe $f_{i}$, all the alternatives can be ranked in descending order. In accordance with closeness coefficient, we can select the best alternative that has value nearest to 1 is nearer to IFPIS $A l t^{*}$ and farther from IFNIS $A l t^{-}$.

The algorithm for the proposed model of TOPSIS is given as follows

\section{Case Study}

4.1. Algorithm for the extended TOPSIS. The Algorithm of Extended TOPSIS is given below as well as in Figure 2.

Step 1: Construct a decision-maker group for the solution of particular problems, then describe the criteria for such a problem. 
Step 2: The decision-makers will select the suitable linguistic variables for the weight of the described criteria and as well as for the ratings of each alternative corresponds to described criteria.

Step 3: Calculate ratings of alternatives and weight of all criteria using (3.2) and (3.3) to get the aggregated rating $\breve{g}_{i j}$ of alternative $A l t_{i}$ corresponds to criteria $\mathrm{Cri}_{j}$ and the aggregated weight $\breve{w}_{j}$ of criteria $\mathrm{Cri}_{j}$.

Step 4: Construct the IF decision matrix after the evaluation of aggregated rating $\breve{g}_{i j}$ of alternative, and aggregated weight $\breve{w}_{j}$ of criteria.

Step 5: Normalize the IF decision matrix by using (3.4) and (3.5) .

Step 6: Form the weighted normalized IF decision matrix by using (3.6).

Step 7: Determine the IFPIS $A l t^{*}$ and IFNIS $A l t^{-}$.

Step 8: Measure the separation of all alternative individually from IFPIS as $\breve{S}^{*}$ and IFNIS as $\breve{S}^{-}$by (3.7) and (3.8), respectively.

Step 9: Compute the closeness coefficient C.Coe $f_{i}$ of all alternative $A l t_{i}$ by (3.9).

Step 10: Arrange all alternatives in the descending order for the selection of ideal solution to the particular problem.
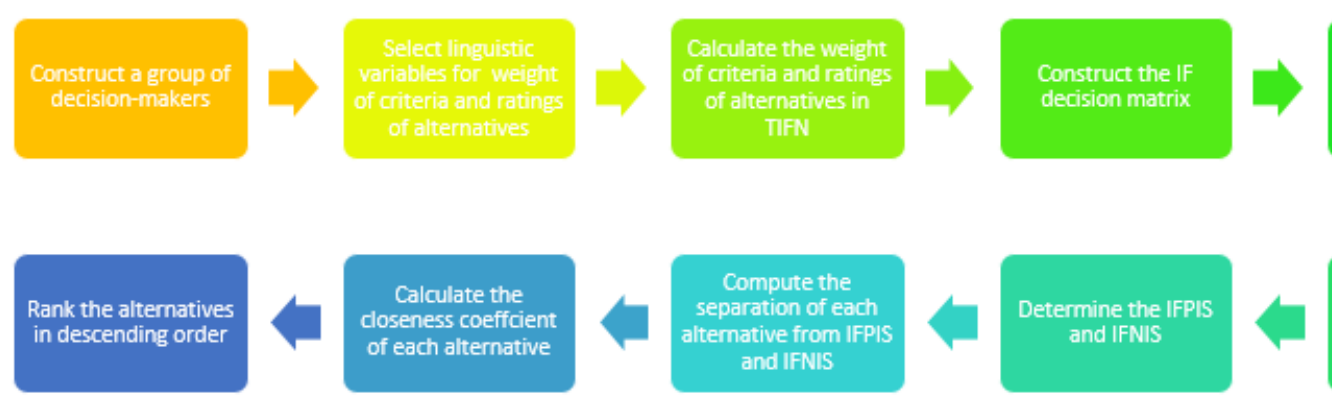

FIGURE 2. Pictorial view of the algorithm of extended TOPSIS

4.2. Numerical Example. To express the procedure of the proposed algorithm, an example from [6] is considered. Suppose a software company has a job offer of system analysis engineer, and they want to select an ideal candidate for the particular job. They made a group of three decision-makers $\breve{D_{1}}, \breve{D}_{2}$ and $\breve{D_{3}}$ for the selection process of the best candidate. Three applicants $A l t_{1}, A l t_{2}$, and $A l t_{3}$ came up for the particular job, and a group of decision-makers interviews them under various criteria. Following are the five benefit criteria that are taken into consideration for the particular job:

- Emotional steadiness $\left(\mathrm{Cri}_{1}\right)$

- Oral communication skill $\left(\mathrm{Cr} i_{2}\right)$

- Personality $\left(\mathrm{Cri}_{3}\right)$

- Past experience $\left(\mathrm{Cri}_{4}\right)$

- Self Confidence $\left(\mathrm{Cri}_{5}\right)$

Methodology of proposed model is applied in following steps to solve such particular problems of decision making. 
Step 1: The group of decision-makers utilize the linguistic variables (Table 1) for the evaluation of weights of various criteria as shown in Table 3.

\begin{tabular}{llll}
\hline & $\breve{D}_{1}$ & $\breve{D}_{2}$ & $\breve{D}_{3}$ \\
\hline$C r i_{1}$ & $\mathrm{H}$ & $\mathrm{VH}$ & $\mathrm{MH}$ \\
$C r i_{2}$ & $\mathrm{VH}$ & $\mathrm{VH}$ & $\mathrm{VH}$ \\
$C r i_{3}$ & $\mathrm{VH}$ & $\mathrm{H}$ & $\mathrm{H}$ \\
$C_{r} i_{4}$ & $\mathrm{VH}$ & $\mathrm{VH}$ & $\mathrm{VH}$ \\
$\mathrm{Cri}_{5}$ & $\mathrm{M}$ & $\mathrm{MH}$ & $\mathrm{MH}$ \\
\hline
\end{tabular}

Table 3 : Evaluation of weights of each criterion $\mathrm{Cri}_{j}$ in LV by decision makers

Step 2: Then decision-makers utilize the linguistic variables (in Table 2) for the evaluation of ratings of each alternative corresponds to each criteria by taking interview of each alternative, and it is shown as in Table 4.

\begin{tabular}{clccc}
\hline Criteria & Alternatives & & Decision Makers \\
& & $\breve{D}_{1}$ & $\breve{D}_{2}$ & $\breve{D}_{3}$ \\
\hline \multirow{3}{*}{$C r i_{1}$} & $A l t_{1}$ & MG & G & MG \\
& $A l t_{2}$ & $\mathrm{G}$ & $\mathrm{G}$ & $\mathrm{MG}$ \\
& $A l t_{3}$ & $\mathrm{VG}$ & $\mathrm{G}$ & $\mathrm{F}$ \\
\hline \multirow{3}{*}{$C r i_{2}$} & $A l t_{1}$ & $\mathrm{G}$ & $\mathrm{MG}$ & $\mathrm{F}$ \\
& $A l t_{2}$ & $\mathrm{VG}$ & $\mathrm{VG}$ & $\mathrm{VG}$ \\
& $A l t_{3}$ & $\mathrm{MG}$ & $\mathrm{G}$ & $\mathrm{VG}$ \\
\hline \multirow{3}{*}{$C r i_{3}$} & $A l t_{1}$ & $\mathrm{~F}$ & $\mathrm{G}$ & $\mathrm{G}$ \\
& $A l t_{2}$ & $\mathrm{VG}$ & $\mathrm{VG}$ & $\mathrm{G}$ \\
& $A l t_{3}$ & $\mathrm{G}$ & $\mathrm{MG}$ & $\mathrm{VG}$ \\
\hline \multirow{3}{*}{$C r i_{4}$} & $A l t_{1}$ & $\mathrm{VG}$ & $\mathrm{G}$ & $\mathrm{VG}$ \\
& $A l t_{2}$ & $\mathrm{VG}$ & $\mathrm{VG}$ & $\mathrm{VG}$ \\
& $A l t_{3}$ & $\mathrm{G}$ & $\mathrm{VG}$ & $\mathrm{MG}$ \\
\hline \multirow{2}{*}{$C r i_{5}$} & $A l t_{1}$ & $\mathrm{~F}$ & $\mathrm{~F}$ & $\mathrm{~F}$ \\
& $A l t_{2}$ & $\mathrm{VG}$ & $\mathrm{MG}$ & $\mathrm{G}$ \\
& $A l t_{3}$ & $\mathrm{G}$ & $\mathrm{G}$ & $\mathrm{MG}$ \\
\hline
\end{tabular}

Table 4 : Evaluation of ratings for each alternative $A l t_{i}$ correspond to criteria $C r i_{j}$ in LV by decision-makers

Step 3: Using Table 1 and Table 2 for the transformation of linguistic variables into TIFNs. The linguistic assessments (as shown in Tables 3 and 4) are converted in TIFNs, and then aggregated ratings of alternatives and aggregated weights for each criteria can be calculated by (3.2) and (3.3), respectively.

Step 4: The IF decision matrix is formed after the evaluation of aggregated ratings of alternatives and aggregated weights of criteria as shown in Table 5.

Step 5: By using linear scale transformation (3.4), the normalized IF decision matrix is obtained as Table 6 . 


\begin{tabular}{llllll}
\hline & $\mathrm{Cri}_{1}$ & $\mathrm{Cri}_{2}$ & $\mathrm{Cri}_{3}$ & $\mathrm{Cri}_{4}$ & $\mathrm{Cri}_{5}$ \\
\hline Alt $_{1}$ & $(5.67,7.67,9.33)$, & $(5,7,8.67)$, & $(5.67,7.67,9)$, & $(8.33,9.67,10)$, & $(3,5,7)$, \\
& $(5,7.67,9.67)$ & $(4.17,7,9.17)$ & $(4.67,7.67,9.33)$ & $(7.33,9.67,10)$ & $(2,5,8)$ \\
\hline Alt $_{2}$ & $(6.33,8.33,9.67)$, & $(9,10,10)$, & $(8.33,9.67,10)$, & $(9,10,10)$, & $(7,8.67,9.67)$, \\
& $(5.5,8.33,9.83)$ & $(8,10,10)$ & $(7.33,9.67,10)$ & $(8,10,10)$ & $(6.17 .8 .67,9.83)$ \\
\hline Alt $_{3}$ & $(6.33,8,9)$, & $(7,8.67,9.67)$, & $(7,8.67,9.67)$, & $(7,8.67,9.67)$, & $(6.33,8.33,9.67)$, \\
& $(5.33,8,9.33)$ & $(6.17 .8 .67,9.83)$ & $(6.17 .8 .67,9.83)$ & $(6.17 .8 .67,9.83)$ & $(5.5,8.33,9.83)$ \\
\hline \multirow{2}{*}{ Weight $(0.7,0.867,0.967)$} & $(0.9,1,1)$ & $(0.767,0.933,1)$ & $(0.9,1,1)$ & $(0.433,0.633,0.833)$ \\
& $(0.617,0.867,0.983)(0.8,1,1)$ & $(0.67,0.933,1)$ & $(0.8,1,1)$ & $(0.367,0.633,0.9)$ \\
\hline
\end{tabular}

Table 5 : IF decision matrix

\begin{tabular}{cllll}
\hline $\mathrm{Cri}_{1}$ & $\mathrm{Cri}_{2}$ & $\mathrm{Cri}_{3}$ & $\mathrm{Cri}_{4}$ & $\mathrm{Cri}_{5}$ \\
\hline $\mathrm{Alt}_{1}(0.59,0.79,0.96),(0.5,0.7,0.86)$, & $(0.57,0.77,0.9)$, & $(0.83,0.97,1)$, & $(0.31,0.52,0.72)$, \\
$(0.51,0.65,1)$ & $(0.45,0.59,1)$ & $(0.5,0.6,1)$ & $(0.61,0.64,0.84)$ & $(0.25,0.4,1)$ \\
\hline Alt $_{2}(0.65,0.86,1)$, & $(0.9,1,1)$, & $(0.83,0.97,1)$, & $(0.9,1,1)$, & $(0.72,0.89,1)$, \\
$(0.5,0.6,0.9)$ & $(0.41,0.41,0.52)$ & $(0.46,0.48,0.63)$ & $(0.61,0.61,0.77)$ & $(0.2,0.23,0.32)$ \\
\hline Alt $_{3}(0.65,0.83,0.93),(0.7,0.87,0.97)$, & $(0.7,0.87,0.97)$, & $(0.7,0.87,0.97)$, & $(0.65,0.86,1)$, \\
$(0.53,0.62,0.93)$ & $(0.42,0.48,0.67)$ & $(0.47,0.54,0.76)$ & $(0.62,0.71,1)$ & $(0.2,0.24,0.36)$ \\
\hline
\end{tabular}

Table 6 : Normalized IF decision matrix

\begin{tabular}{cllll}
\hline $\mathrm{Cri}_{1}$ & \multicolumn{1}{c}{$\mathrm{Cri}_{2}$} & $\mathrm{Cri}_{3}$ & $\mathrm{Cri}_{4}$ & $\mathrm{Cri}_{5}$ \\
\hline Alt $_{1}(0.41,0.68,0.92),(0.45,0.7,0.86)$, & $(0.43,0.71,0.9)$, & $(0.74,0.97,1)$, & $(0.13,0.33,0.6)$, \\
$(0.31,0.56,0.98)$ & $(0.36,0.59,1)$ & $(0.33,0.56,1)$ & $(0.49,0.64,0.84)$ & $(0.09,0.25,0.9)$ \\
\hline Alt $_{2}(0.45,0.74,0.97),(0.81,1,1)$, & $(0.63,0.9,1)$, & $(0.81,1,1)$, & $(0.31,0.56,0.9)$, \\
$(0.31,0.52,0.88)$ & $(0.32,0.41,0.52)$ & $(0.30,0.45,0.63)$ & $(0.49,0.61,0.77)$ & $(0.07,0.14,0.29)$ \\
\hline Alt $_{3}(0.45,0.72,0.9)$, & $(0.63,0.87,0.97),(0.53,0.81,0.97)$, & $(0.63,0.87,0.97),(0.28,0.54,0.83)$, \\
$(0.33,0.54,0.91)$ & $(0.34,0.48,0.67)$ & $(0.31,0.5,0.76)$ & $(0.5,0.71,1)$ & $(0.07,0.15,0.32)$ \\
\hline
\end{tabular}

Table 7 : Weighted normalized IF decision matrix

Step 6: The weighted normalized IF decision matrix can be obtained by (3.6) as shown in Table 7.

Step 7: The IFPIS $A l t^{*}$ and IFNIS $A l t^{-}$is determined as $A l t^{*}=[((1,1,1),(0,0,0)),((1,1,1),(0,0,0)),((1,1,1),(0,0,0)),((1,1,1),(0,0,0)),((1,1,1),(0,0,0))]$ $A l t^{-}=[((0,0,0),(1,1,1)),((0,0,0),(1,1,1)),((0,0,0),(1,1,1)),((0,0,0),(1,1,1)),((0,0,0),(1,1,1))]$

Step 8: Calculating the separation of all alternative from IFPIS $A l t^{*}$ and IFNIS $A l t^{-}$by using (3.7) and (3.8), respectively, as shown in Table 8.

\begin{tabular}{lll}
\hline & $\breve{S}^{*}$ & $\breve{S}^{-}$ \\
\hline$A l t_{1}$ & 2.7659 & 3.0305 \\
\hline Alt $_{2}$ & 2.0025 & 3.6077 \\
\hline Alt $_{3}$ & 2.3098 & 3.3237
\end{tabular}

Table 8 : Separation of alternatives from IFPIS and IFNIS

Step 9: The closeness coefficient C.Coe $f_{i}$ of alternatives is calculated by (3.9) as shown in Table 9. 


\begin{tabular}{lll}
\hline & C.Coef & Rank \\
\hline Alt $_{1}$ & 0.522 & 3 \\
\hline Alt $_{2}$ & 0.643 & 1 \\
\hline Alt $_{3}$ & 0.589 & 2
\end{tabular}

Table 9 : Closeness coefficient of alternatives and ranking of alternatives

Step 10: Rank the alternatives by the evaluation of According to the closeness coefficient C.Coe $f_{i}$ in descending order from highest to lowest as shown in Table 9. We can determine the best candidate for particular job from Table 9, and according to our proposed model $A l t_{2}$ is the best selection.

\section{Comparative Study}

The results are compared with several papers [6, 7, 12, 18, 13], where different extensions of TOPSIS techniques for the decision making were applied in the fuzzy, intuitionistic fuzzy, and type-2 fuzzy environments. Here the set of alternatives and criteria are kept the same purposely, and as well as the evaluation of weights of criteria and ratings of alternatives by decision-makers are kept the same purposely. Chen [6] applied extension of TOPSIS on fuzzy structure, while Chu et al. [7] has overcome with improvement in Chen's model. Mahdavi et al. [18] proposed a new extension of fuzzy TOPSIS and found the same results with Chen and Ta-Chung. Gautam et al. [13] proposed the extension of TOPSIS in intuitionistic fuzzy structure, and their findings were different from the others, while we proposed a novel model of extended TOPSIS on intuitionistic fuzzy structure, where the results are matched with $[6,7,18]$. The proposed model of extended TOPSIS in this article is far better than Gautaum's model since we have the same results with other authors' models. Ghaemi et al. [12] applied TOPSIS technique on type-2 fuzzy set. Table 10 shows a comparison of the ordering of alternatives corresponds to different articles. If we compare the result, that shows $A l t_{2}$ is the best alternative under our proposed model and other authors' models, whereas $A l t_{1}$ is the worst alternative.

\begin{tabular}{lr}
\hline Different method of TOPSIS & Ordering of alternatives \\
\hline Chen [24] & $A l t_{2} \succ A l t_{3} \succ A l t_{1}$ \\
Chu et al. [25] & $A l t_{2} \succ A l t_{3} \succ A l t_{1}$ \\
Ghaemi et al. [26] & $A l t_{2} \succ A l t_{1} \succ A l t_{3}$ \\
Mahdavi et al. [27] & $A l t_{2} \succ A l t_{3} \succ A l t_{1}$ \\
Gautam et al. [28] & $A l t_{3} \succ A l t_{1} \succ A l t_{2}$ \\
Proposed Model & $A l t_{2} \succ A l t_{3} \succ A l t_{1}$ \\
\hline
\end{tabular}

Table 10 : Comparison of results to other articles

\section{Conclusion and Future Work}

In practical life, multi-criteria problems contain uncertain, vague, and complex data. To deal with such data, fuzzy set theory is an appropriate structure. But this concept is further extended to intuitionistic fuzzy set theory to study the uncertain data in deep. In this article, 
an extension of TOPSIS is proposed to Chen's model [24], which deals with the linguistic evaluation of decision-makers to tackle the multi-criteria decision-making (MCDM) issues in the light of intuitionistic fuzzy structure. Linguistic variables are used mostly as compare to numeric values in real-life while taking any decision, so on the evaluation of linguistic variables, this proposed model selects the best alternative. The representation of linguistic variables is analyzed by triangular Intuitionistic fuzzy numbers (TIFNs). A new method for distance measure is introduced between two TIFNs which is simple and more effective. A new concept is given to determine intuitionist fuzzy positive solution (IFPIS) and intuitionistic negative ideal solution (IFNIS). Also, the distance measure is used to quantify the separation of alternatives from the desired IFPIS and the desired IFNIS for the sake of the best alternative. Chen's example [24] of a personal selection concern is discussed. In the comparative analysis with other articles based on linguistic variables evaluation, it can be seen that the previously proposed TOPSIS technique for TIFN by Gautaum [13] gave different results with other models of TOPSIS, while this model has the same result, which concludes that this technique is far better. Generalization of TFN to TIFN gave the depth study of the data than fuzzy environment, which gives the appropriate results in decision making with the membership and non-membership function degree. The use of linguistic variables as an evaluation method makes calculation and decision easy for the decisionmakers committee. However, this method can also be applicable in medical drug selection for pandemic disease, selecting players for an upcoming important series of matches, and more problems regarding strategic decisions. Moreover, this TOPSIS technique can be further extended to trapezoidal, octagonal, and decagonal fuzzy and intuitionistic fuzzy numbers to study the data in more depth for the best result.

\section{REFERENCES}

[1] K.T. Atanassov, Intuitionistic fuzzy sets, Fuzzy Sets and Systems, 20, No. 1 (1986) 87-96.

[2] K.T. Atanassov, G. Gargov, Interval-valued intuitionistic fuzzy sets, Fuzzy Sets and Systems, 31, No. 3 (1989) 343-349.

[3] G. Atalik, S. Senturk, A noval ranking approach based on incircle of triangular intuitionistic fuzzy numbers, Journal of Intelligent \& Fuzzy Systems, 39, No. 5 (2020) 6271-6278.

[4] G. Atalik, S. Senturk, A New Ranking Method for Triangular Intuitionistic Fuzzy Number based on Gergonne Point, Nicel Bilimler Dergisi, 1, No. 1 (2019) 59-73.

[5] P. Burillo, H. Bustince, V. Mohedano, Some definitions of intuitionistic fuzzy number. First properties, In Proceedings in Fuzzy Based Expert Systems, (1994) 53-55.

[6] C.T. Chen, Extensions of the TOPSIS for group decision-making under fuzzy environment, Fuzzy Sets and Systems, 114, No. 1 (2000) 1-9.

[7] T.C. Chu, Y.C Lin, Improved extensions of the TOPSIS for group decisionmaking under fuzzy environment, Journal of Information and Optimization Sciences, 23, No. 2 (2002) 273-286.

[8] D. Dubois, H. Prade, Fuzzy numbers: an overview, Readings in Fuzzy Sets for Intelligent Systems, (1993) 112-148.

[9] R. Fuller, P. Majlender, On weighted possibilistic mean and variance of fuzzy numbers, Fuzzy Sets and Systems, 136, No. 3 (2003) 363-374.

[10] V.S. Gadakh, Parametric optimization of wire electrical discharge machining using TOPSIS method, Advances in Production Engineering \& Management, 7, No. 3 (2012) 157-164.

[11] A.S. Garge, S.A. Shirali, Triangular numbers, Resonance, 17, No. 7 (2012) 672-681.

[12] N.F. Ghaemi, M.M. Rostamy, Extension of TOPSIS for group decision-making based on the type-2 fuzzy positive and negative ideal solutions, International Jouranl of Industrial Mathematics, 2, No. 3 (2010) 199213. 
[13] S.S. Gautam, S.R. Singh, TOPSIS for multi criteria decision making in intuitionistic fuzzy environment, International Journal of Computer Applications, 156, No. 8 (2016) 42-49.

[14] H.M. Hsu, C.T. Chen, Fuzzy hierarchical weight analysis model for multicriteria decision problem, Journal of the Chinese Institute of Industrial Engineers, 11, No. 3 (1994) 126-136.

[15] C.L. Hwang, Y.J. Lai, T.Y. Liu, A new approach for multiple objective decision making, Computers \& operations research, 20, No. 8 (1993) 889-899.

[16] Y.J. Lai, T.Y. Liu, C.L. Hwang, TOPSIS for MODM, European Journal of Operational Research, 76, No. 3 (1994) 486-500.

[17] D.F. Li, J.X. Nan, M.J. Zhang, A ranking method of triangular intuitionistic fuzzy numbers and application to decision making, International Journal of Computational Intelligence Systems, 3, No. 5 (2010) 522-530.

[18] I. Mahdavi, N. Mahdavi-Amiri, A. Heidarzade, Designing a model of fuzzy TOPSIS in multiple criteria decision making, Applied Mathematics and Computation, 206, No. 2 (2008) 607-617.

[19] K. Naeem, M. Riaz, X. Peng, D. Afzal, Pythagorean fuzzy soft MCGDM methods based on TOPSIS, VIKOR and aggregation operators, Journal of Intelligent \& Fuzzy Systems 37, No. 5 (2019) 6937-6957.

[20] K. Naeem, M. Riaz, D. Afzal, Pythagorean m-polar Fuzzy Sets and TOPSIS method for the Selection of Advertisement Mode, Journal of Intelligent \& Fuzzy Systems 37, No. 6 (2019) 8441-8458.

[21] H.M. Nehi, A new ranking method for intuitionistic fuzzy numbers, International Journal of Fuzzy Systems, 12, No. 1 (2010) 80-86.

[22] Y. Qin, X. Cui, M. Huang, Y. Zhong, Z. Tang, P. Shi, Linguistic Interval-Valued Intuitionistic Fuzzy Archimedean Power Muirhead Mean Operators for Multiattribute Group Decision-Making, Complexity, 2020, (2020) 2373762.

[23] M. Saeed, M. Saqlain, A. Mehmood, K. Naseer, S. Yaqoob, MultiPolar Neutrosophic Soft Sets with Application in Medical Diagnosis and Decision-Making, Neutrosophic Sets and Systems, 33, No. 1 (2020) 183-207.

[24] M. Saeed, M. Saqlain, M. Riaz, Application of Generalized Fuzzy TOPSIS in Decision Making for Neutrosophic Soft set to Predict the Champion of FIFA 2018: A Mathematical Analysis, Punjab University Journal of Mathematics, 51, No. 8 (2019) 111-126.

[25] M. Saeed, M. Saqlain, A. Mehmood, Application of Similarity Measure on m-polar Interval-valued Neutrosophic Set in Decision Making in Sports, Neutrosophic Sets and Systems, 38, No. 1 (2020) 317-334.

[26] R.H.N. Santiago, C. Maeder, Linguistic Variables of Type-N. A Mathematical Model, TEMA-Tendencias em Matematica Aplicada e Computacional, 12, No. 1 (2011) 21-30.

[27] M.R. Seikh, P.K. Nayak, M. Pal, Notes on triangular intuitionistic fuzzy numbers, International Journal of Mathematics in Operational Research, 5, No. 4 (2013) 446-465.

[28] M. Saqlain, M. Saeed, M.R. Ahmad, F. Smarandache, Generalization of TOPSIS for Neutrosophic Hypersoft set using Accuracy Function and its Application, Neutrosophic Sets and Systems, 27, No. 1 (2019) 131-137.

[29] M. Saqlain, N. Jafar, R. Hamid A. Shahzad, Prediction of Cricket World Cup 2019 by TOPSIS Technique of MCDM-A Mathematical Analysis, International Journal of Scientific \& Engineering Research, 10, No. 2 (2019) 789-792.

[30] Z.B. Wu, Y.H. Chen, The maximizing deviation method for group multiple attribute decision-making under linguistic environment, Fuzzy Sets and Systems, 158, No. 14 (2007) 1608-1617.

[31] Y.J. Xu, Some New Operations on Triangular Fuzzy Number Intuitionistic Fuzzy Set, 2019 Chinese Control And Decision Conference (CCDC), (2019) 3760-3763.

[32] Z.L. Yue, An extended TOPSIS for determining weights of decision-makers with interval numbers, Knowledge-based Systems, 24, No. 1 (2011) 146-153.

[33] K.P. Yoon, A reconciliation among discrete compromise solutions, Journal of the Operational Research Society, 38, No. 3 (1987) 277-286.

[34] K. Yoon, C.L. Hwang, Multiple Attribute Decision Making, Methods and Applications A State-of-the-Art Survey, Springer, 1981.

[35] L.A. Zadeh, Fuzzy sets, Infromation and Control, 8, No. 3 (1965) 338-353.

[36] L.A. Zadeh, The concept of a linguistic variable and its application to approximate reasoning-I, Information Sciences, 8, No. 3 (1975) 199-249.

[37] F. Zafar, M. Akram, A novel decision-making method based on rough fuzzy information, International Journal of Fuzzy Systems, 20, No. 3 (2018) 1000-1014. 
[38] S.H. Zanakis, A. Solomon, N. Wishart, S. Dublish, Multi-attribute decision making: A simulation comparison of select methods, European Journal of Operational Research, 107, No. 3 (1998) 507-529.

[39] M. Zulqarnain, F. Dayan, M. Saeed, TOPSIS analysis for the prediction of diabetes based on general characteristics of humans, International Journal of Pharmaceutical Sciences and Research, 9, No. 7 (2018) 29322939.

[40] R.M. Zulqarnain, M. Saeed, B. Ali, N. Ahmad, L. Ali, Application of Interval Valued Fuzzy Soft Max-Min Decision Making Method, International Journal, 9, No. 1 (2020) 11-19. 